\title{
A silicon photomultiplier readout for time of flight neutron spectroscopy with $\gamma$-ray detectors
}

\author{
A. Pietropaolo, ${ }^{1}$ G. Gorini, ${ }^{1}$ G. Festa ${ }^{2, a)}$ C. Andreani, ${ }^{2}$ M. P. De Pascale, ${ }^{2}$ E. Reali, ${ }^{2}$ \\ F. Grazzi, ${ }^{3}$ and E. M. Schooneveld ${ }^{4}$ \\ ${ }^{1}$ Dipartimento di Fisica "G. Occhialini" and CNISM, Università Degli Studi di Milano-Bicocca, Piazza della \\ Scienza 3, 20126 Milano, Italy \\ ${ }^{2}$ Dipartimento di Fisica and Centro NAST, Università degli Studi di Roma Tor Vergata, Via della Ricerca \\ Scientifica 1, 00133, Roma, Italy \\ ${ }^{3}$ Istituto dei Sistemi Complessi-Consiglio Nazionale delle Ricerche, Via Madonna del Piano n.10, I-50019 \\ Sesto Fiorentino, Firenze, Italy \\ ${ }^{4}$ ISIS Facility, Rutherford Appleton Laboratory, Chilton, Didcot, Oxfordshire, OX11 0QX, United Kingdom
}

(Received 15 July 2009; accepted 4 August 2009; published online 11 September 2009)

\begin{abstract}
The silicon photomultiplier (SiPM) is a recently developed photosensor used in particle physics, e.g., for detection of minimum ionizing particles and/or Cherenkov radiation. Its performance is comparable to that of photomultiplier tubes, but with advantages in terms of reduced volume and magnetic field insensitivity. In the present study, the performance of a gamma ray detector made of an yttrium aluminum perovskite scintillation crystal and a SiPM-based readout is assessed for use in time of flight neutron spectroscopy. Measurements performed at the ISIS pulsed neutron source demonstrate the feasibility of $\gamma$-detection based on the new device. (c) 2009 American Institute of Physics. [doi:10.1063/1.3212677]
\end{abstract}

Silicon photomultipliers (SiPMs) are semiconductor photosensitive devices consisting of an avalanche photodiode matrix (typical pixel size $20-30 \mu \mathrm{m}$ ) on a common silicon substrate. ${ }^{1-4}$ The pixels are electrically decoupled from each other by polysilicon resistors (typical value $500 \mathrm{k} \Omega$ ) located on the same substrate. The operational bias voltage is $10 \%-15 \%$ higher than the breakdown voltage so that each pixel operates in a limited Geiger mode with a gain determined by the charge accumulated in pixel capacitance (typically $100 \mathrm{fF}$ ). In this mode, a photoelectron created in a pixel of the SiPM and reaching the high field region initiates a Geiger discharge confined to that pixel. The pixel discharge is quenched by limiting the current to about $10 \mu \mathrm{A}$ through the polysilicon resistor. The independently operating pixels are connected to the same readout line. The combined output signal is the sum of all the so-called fired pixels, which is a measure of the light flux on the SiPM. Main SiPM advantages compared to conventional photomultiplier tubes (PMTs) are insensitivity to magnetic fields, low operation voltage, and compact size. Moreover, they feature a high peak photon detection efficiency (exceeding 50\% in some cases) and good time response. Their small dimensions allow for the construction of compact, light, and robust detection devices. So far SiPM have been used in Cherenkov detectors, minimum ionizing particles, and dark matter (e.g., neutralino) searches. ${ }^{5-7}$

In the present study we investigate the performance of a compact $\gamma$-ray detector made of an yttrium aluminum perovskite (YAP) scintillator crystal and a SiPM-based readout, for time of flight (TOF) neutron spectroscopy at pulsed neutron sources.

\footnotetext{
a) Author to whom correspondence should be addressed. FAX: +39062023507. Electronic mail: giulia.festa@ roma2.infn.it.
}

TOF neutron spectroscopy is a powerful technique for probing material properties with potential applications in material science, energy, electronics, and cultural heritage research. Among the possible applications, a very interesting one is represented by the neutron resonance capture analysis (NRCA). ${ }^{8}$ This technique exploits the peculiar interactions of the epithermal neutrons $\left(1-10^{4} \mathrm{eV}\right.$ energy) in materials. Several nuclei, especially the heavy metal ones, exhibit resonances in the neutron radiative capture cross section. When a neutron is absorbed at the resonance energy, a prompt $\gamma$-ray cascade is generated following the $(n, \gamma)$ reaction: $n+{ }^{A} X_{Z}$ $\rightarrow{ }^{A+1} X_{Z}^{*} \rightarrow{ }^{A+1} X_{Z}+\sum_{i=1, N} \gamma_{i}$, where ${ }^{A} X_{Z}$ represents the nuclide with atomic mass $A$ and atomic number $Z$. In the first step an excited nucleus is formed which in turn decays to the ground state by multistep transitions. This technique is capable of providing information on the elemental composition of the object, as different elements (as well as different isotopes of the same element) have resonances at different energies. At a pulsed neutron source, the fingerprints of the different elements present within an irradiated object appear as peaks in the TOF spectrum, their time positions being univocally related to the resonance energies following the relation:

$$
t_{\mathrm{res}}=\sqrt{0.5 \frac{m_{n}}{E_{r}}} L_{0},
$$

$m_{n}, E_{r}$, and $L_{0}$ being the neutron mass, the resonance energy, and the neutron flight path, respectively.

The objective of the present experimental investigation is the study of a compact detection system for NRCA experiments relying on a SiPM-based readout for NRCA experiments. To this aim, a dedicated detection system was designed and produced that was composed of a YAP scintillator crystal coupled to a SiPM and a PMT, both from Hamamatsu. ${ }^{9}$ The YAP crystal was $6 \times 6 \times 25 \mathrm{~mm}^{3}$, the 


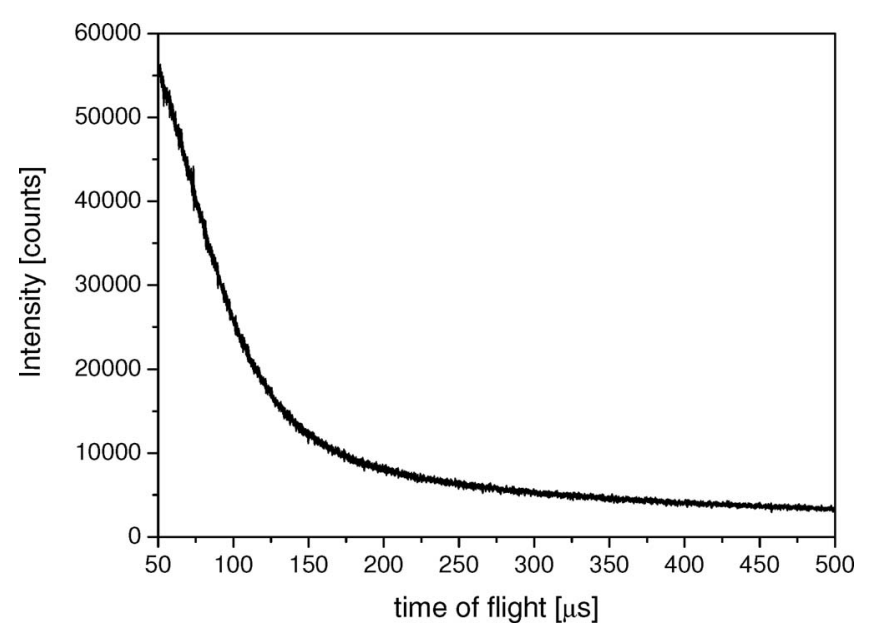

FIG. 1. TOF transmission spectrum recorded on the PEARL beam line inserting a YAP crystal in the incident neutron beam: No dips are found in the spectrum, thus confirming that no resonances are present in the eV-keV energy range. The integrated beam current for the runs is $15.6 \mu \mathrm{A} \mathrm{h}$.

SiPM active area was of $1 \mathrm{~mm}^{2}(25 \mu \mathrm{m}$ pixel size) and the PMT photocathode was about $50 \mathrm{~mm}^{2}$. The use of SiPM and PMT was aimed at a comparative assessment of the effectiveness of the YAP-SiPM device with respect to a standard one. Indeed, YAP detectors and PMTs have been successfully employed for other applications of neutron spectroscopy. ${ }^{10-12}$ YAP is a nonhygroscopic crystal and easy to be machined in different shapes. Its effective atomic number $Z_{\text {eff }}=36$ and density $\rho=5.55 \mathrm{~g} \mathrm{~cm}^{-3}$ provide a good efficiency $(10 \%$ at $1 \mathrm{MeV})$, the appreciable light yields (18000 photons $/ \mathrm{MeV}$ ) a sufficient energy resolution and its fast decay time $(\tau=27 \mathrm{~ns})$ is a reliable use for TOF neutron spectroscopy. ${ }^{10}$ Moreover its sensitivity to neutrons is low. This can be appreciated in Fig. 1, where a TOF spectrum is shown that was recorded by placing a YAP crystal directly in the neutron beam. The absence of dips in the time region shown in the figure, corresponding to an energy range from $0.5 \mathrm{eV}$ to a few $\mathrm{keV}$, is a confirmation of the absence of resonant absorption in the YAP crystal.

The NRCA measurements have been performed on the INES beam line ${ }^{13-15}$ at the ISIS pulsed neutron source. ${ }^{16}$ This beam line is characterized by a white pulsed neutron beam, moderated by a water moderator at $295 \mathrm{~K}$, placed at a distance $L_{0} \approx 23 \mathrm{~m}$ from the sample position. The beam area at the sample position is $4.0 \times 4.0 \mathrm{~cm}^{2}$. The setup for the experiment is schematically shown in Fig. 2.

For the measurements the SiPM was biased with a (stabilized) tension of $71 \mathrm{~V}$ (slightly above the breakdown voltage) thus providing a gain of about $3 \times 10^{5}$. The signal from the SiPM was sent to a purpose-built preamplifier with a fixed (and stabilized) gain of 23. The PMT gain was about $4 \times 10^{5}$. The signal from the SiPM and the PMT was discriminated before being collected by the data acquisition system of ISIS. The lower level discrimination (LLD) threshold of the discriminator was set at about $300 \mathrm{keV}$ (energy equivalent threshold) for both detection systems. The time data recording window ( $20 \mathrm{~ms}$ duration) is started by the ISIS trigger related to the proton pulse $(50 \mathrm{~Hz})$ and the TOF is determined by the detection of the prompt $\gamma$ rays from the sample. The detector signal processing electronics provides a stop signal for the TOF data recording system if the energy

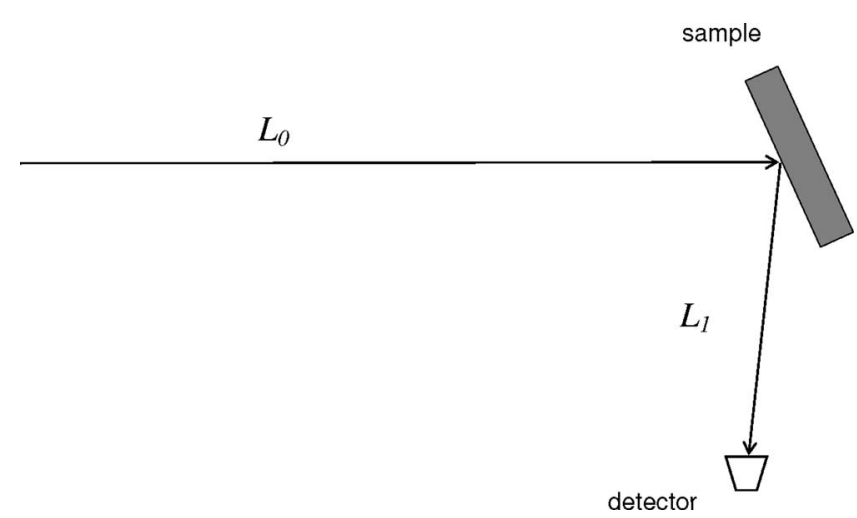

FIG. 2. Layout of the NRCA experiment on the INES beam line.

released in the detector is above the electronic LLD threshold; the time registered by the detector readout electronics is therefore proportional to the resonance energy. In order to produce resonance neutron radiative capture $\gamma$ rays, a slab of $\mathrm{Cu} / \mathrm{Sb}\left(\mathrm{Cu}_{92} \mathrm{Sb}_{8}\right) 5 \mathrm{~mm}$ thick and $20 \mathrm{~cm}^{2}$ surface area was placed at the sample position. Both $\mathrm{Cu}$ and $\mathrm{Sb}$ have several resonances in the neutron radiative capture cross section $\sigma_{\mathrm{RC}}(E)$ in the $\mathrm{eV}$ region, as shown in Fig. 3 where $\sigma_{\mathrm{RC}}(E)$ is plotted for the natural $\mathrm{Cu}$ and $\mathrm{Sb}$. As already mentioned before, the resonance peaks in the $\sigma_{\mathrm{RC}}(E)$ appear in the TOF spectrum at positions that can be precisely calculated through Eq. (1).

Figure 4 shows the normalized TOF spectra recorded by the SiPM and the PMT in the region up to $800 \mu \mathrm{s}$, where the main absorption resonances are found. The average ratio of the two spectra is about 1.5 (varying between about 3.5 and 0.5 at low and high flight times, respectively), as shown in Fig. 4(c). The presence of structures in the intensity ratio is due to the different proportions of peak intensity and background beneath the peaks for the two spectra.

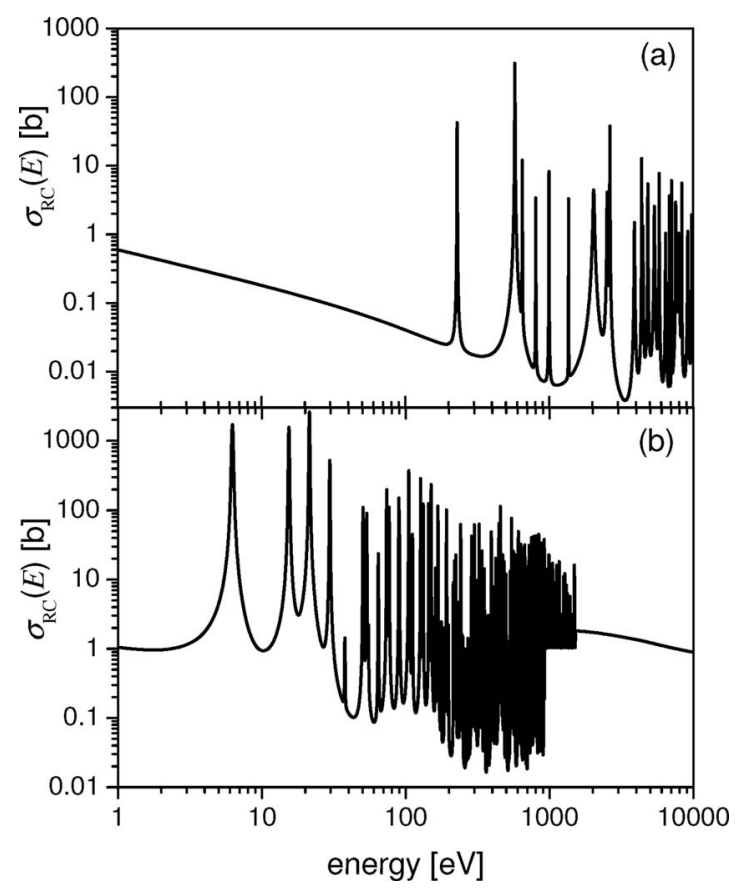

FIG. 3. Radiative capture cross section $\sigma_{\mathrm{RC}}(E)$ for natural $\mathrm{Cu}(\mathrm{a})$ and $\mathrm{Sb}(\mathrm{b})$ in the epithermal energy region. 


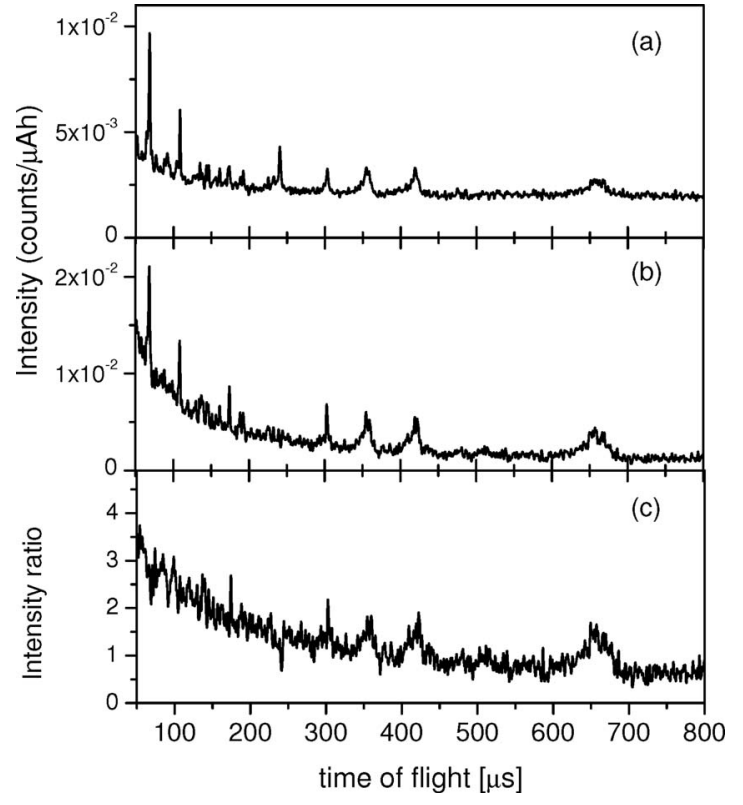

FIG. 4. Neutron TOF spectra recorded by SiPM (a) and PMT (b) with the $\mathrm{Cu}_{92} \mathrm{Sb}_{8}$ absorbing sample. Panel (c) shows the intensity ratio of the spectra in (a) and (b) in the same TOF region.

Table I lists the experimental time positions of the peaks in Fig. 4, together with the corresponding resonance energies $E_{r}$ and isotope assignment. The experimental values of the peaks time positions are in very good agreement within the experimental uncertainties with the values of $t_{\text {res }}$ estimated through Eq. (1) using the tabulated resonance energies of the $\mathrm{Cu}$ and $\mathrm{Sb}$ (see Fig. 3). This allows us to assign each peak to a given isotopic species of both $\mathrm{Cu}$ and $\mathrm{Sb}$. As far as the full width at half maximum (FWHM) is concerned, it can be noted that for the main peaks observed in Fig. 4, the corresponding FWHMs are comparable for all the peaks considered, with differences between $10 \%$ and $20 \%$ (see Table II).

The peak to background ratio for the YAP-PMT system is between 0.9 and 2.0 for the peaks found in the time region above $300 \mu$ s, while it is 0.6 and 0.9 for the main two peaks below $120 \mu$ s. For the YAP-SiPM device, this ratio is between 0.35 and 0.60 in the former interval, and 1.15 and 1.82

TABLE I. TOF positions of the resonance peaks in the two spectra of Figs. 4(a) and 4(b) compared to the time positions $t_{\text {res }}$ calculated through Eq. (1). The calculated uncertainties for $t_{\text {res }}$ are in the order of $1 \mu \mathrm{s}$. The last two columns list the corresponding resonance energies and isotope assignment.

\begin{tabular}{cccrc}
\hline \hline $\begin{array}{c}\mathrm{TOF}_{\text {PMT }} \\
(\mu \mathrm{s})\end{array}$ & $\begin{array}{c}\mathrm{TOF}_{\text {SiPM }} \\
(\mu \mathrm{s})\end{array}$ & $\begin{array}{c}t_{\text {res }} \\
(\mu \mathrm{s})\end{array}$ & $\begin{array}{c}E_{r} \\
(\mathrm{eV})\end{array}$ & ${ }^{A} X$ \\
\hline $662.02 \pm 0.26$ & $662.53 \pm 0.26$ & 663.90 & 6.16 & ${ }^{121} \mathrm{Sb}$ \\
$420.67 \pm 0.26$ & $419.67 \pm 0.26$ & 420.10 & 15.37 & ${ }^{121} \mathrm{Sb}$ \\
$354.36 \pm 0.26$ & $355.04 \pm 0.26$ & 354.80 & 21.59 & ${ }^{123} \mathrm{Sb}$ \\
$302.67 \pm 0.26$ & $303.19 \pm 0.26$ & 306.60 & 28.85 & ${ }^{121} \mathrm{Sb}$ \\
$225.49 \pm 0.26$ & $225.32 \pm 0.26$ & 226.20 & 53.09 & ${ }^{121} \mathrm{Sb}$ \\
$203.27 \pm 0.26$ & $204.65 \pm 0.26$ & 205.30 & 64.26 & ${ }^{121} \mathrm{Sb}$ \\
$182.43 \pm 0.26$ & $182.25 \pm 0.26$ & 183.20 & 77.73 & ${ }^{123} \mathrm{Sb}$ \\
$161.07 \pm 0.26$ & $160.89 \pm 0.26$ & 160.30 & 105.50 & ${ }^{123} \mathrm{Sb}$ \\
$119.21 \pm 0.26$ & $119.37 \pm 0.26$ & 118.40 & 193.62 & ${ }^{123} \mathrm{Sb}$ \\
$108.36 \pm 0.26$ & $108.35 \pm 0.26$ & 108.90 & 228.65 & ${ }^{65} \mathrm{Cu}$ \\
$68.39 \pm 0.26$ & $68.39 \pm 0.26$ & 68.20 & 583.30 & ${ }^{63} \mathrm{Cu}$ \\
$64.43 \pm 0.26$ & $64.26 \pm 0.26$ & 65.10 & 640.66 & ${ }^{63} \mathrm{Cu}$ \\
\hline \hline
\end{tabular}

TABLE II. FWHM for the main peaks in Fig. 4 recorded by SiPM and PMT readouts. Time positions of the resonances $t_{\text {res }}$ determined by Eq. (1) and corresponding isotope specie assignment are also indicated.

\begin{tabular}{cccc}
\hline \hline $\begin{array}{c}t_{\text {res }} \\
(\mu \mathrm{s})\end{array}$ & ${ }^{A} X$ & $\begin{array}{c}\mathrm{FWHM}_{\text {PMT }} \\
(\mu \mathrm{s})\end{array}$ & $\begin{array}{c}\mathrm{FWHM}_{\text {SiPM }} \\
(\mu \mathrm{s})\end{array}$ \\
\hline 663.90 & ${ }^{121} \mathrm{Sb}$ & 25.40 & 26.00 \\
420.10 & ${ }^{121} \mathrm{Sb}$ & 9.65 & 9.64 \\
354.80 & ${ }^{123} \mathrm{Sb}$ & 9.65 & 8.62 \\
306.60 & ${ }^{121} \mathrm{Sb}$ & 2.62 & 2.01 \\
108.90 & ${ }^{65} \mathrm{Cu}$ & 1.37 & 1.36 \\
68.20 & ${ }^{63} \mathrm{Cu}$ & 3.11 & 2.60 \\
\hline \hline
\end{tabular}

for the other one. This difference is due to (i) the intrinsic noise of the SiPM (e.g., thermal noise) especially in the high TOF region; and (ii) to a not completely optimized threshold for YAP-PMT, especially at low TOF. ${ }^{17}$

In summary, a NRCA experiment has been carried out at the INES beam line at ISIS spallation neutron source, to assess the potential of a compact detection device composed of a YAP crystal coupled to a SiPM-based readout. This has been done by detecting resonance neutron radiative capture $\gamma$ rays produced in $(n, \gamma)$ reactions in a $\mathrm{Cu} / \mathrm{Sb}$ sample. The measurement allowed to reliably recognize the main absorption resonances in the composite sample and thus an elemental analysis of the material.

The performance of the SiPM-based device may be improved in terms of counting efficiency and $S / B$ enhancement. The results obtained show the effectiveness and the high potential of the SiPM technology for designing and producing compact, robust, and cheap detectors for TOF neutron spectroscopy at spallation neutron sources, of which NRCA is the specific application investigated in this paper.

This work was supported by the CNR-CCLRC Agreement No. 01/9001 concerning collaboration in scientific research at the spallation neutron source ISIS. The financial support of the Consiglio Nazionale delle Ricerche in this research is hereby acknowledged.

${ }^{1}$ G. Bondarenko, B. Dolgoshein, V. Golovin, A. Ilyin, R. Klanner, and E. Popova, Nucl. Phys. B (Proc. Suppl.) 61B, 347 (1998).

${ }^{2}$ G. Bondarenko, P. Buzhan, B. Dolgoshein, V. Golovin, E. Guschin, A. Ilyin, V. Kaplin, A. Karakash, R. Klanner, V. Pokachalov, E. Popova, and K. Smirnov, Nucl. Instrum. Methods Phys. Res. A 442, 187 (2000).

${ }^{3}$ P. Buzhan, B. Dolgoshein, L. Filatov, A. Ilyin, V. Kantzerov, V. Kaplin, A. Karakash, F. Kayumov, S. Klemin, A. Pleshko, E. Popova, S. Smirnov, and Yu. Volkov, Proceedings of the Seventh International Conference on Advance Technology and Particle Physics, 2002 (unpublished), p. 717.

${ }^{4}$ F.P. Buzhan, B. Dolgoshein, L. Filatov, A. Ilyin, V. Kantzerov, V. Kaplin, A. Karakash, F. Kayumov, S. Klemin, E. Popova, and S. Smirnov, Nucl. Instrum. Methods Phys. Res. A 504, 48 (2003); B. Dolgoshein, V. Balagura, P. Buzhan, M. Danilov, L. Filatov, E. Garutti, M. Groll, A. Ilyin, V. Kantserov, V. Kaplin, A. Karakash, F. Kayumov, S. Klemin, V. Korbel, H. Meyer, R. Mizuk, V. Morgunov, E. Novikov, P. Pakhlov, E. Popova, V. Rusinov, F. Sefkow, E. Tarkovsky, I. Tikhomirov, and Claice/ SiPM Collaboration, ibid. 563, 368 (2006); D. Renker, ibid. 567, 48 (2006).

${ }^{5}$ A. Heering, J. Rohlf, J. Freeman, S. Los, S. Kuleshov, and Y. Musienko, Nucl. Instrum. Methods Phys. Res. A 576, 341 (2007).

${ }^{6}$ S. Korpar, R. Dolenec, K. Hara, T. Iijima, P. Križan, Y. Mazuka, R. Pestotnik, A. Stanovnik, and M. Yamaoka, Nucl. Instrum. Methods Phys. Res. A 595, 161 (2008); R. Pestotnik, R. Dolenec, S. Korpar, P. Križan, and A. Petelin, ibid. 581, 457 (2007).

${ }^{7}$ E. Aprile, P. Cushman, K. Ni, and P. Shagin, Nucl. Instrum. Methods 
Phys. Res. A 556, 215 (2006).

${ }^{8}$ H. Postma and P. Schillebeeckx, Not. Neutroni Luce Sincrotrone 11, 2 (2006); H. Postma, P. Schillebeeckx, and R. B. Halbertsma, Archaeometry 46, 635 (2004).

${ }^{9}$ Official website of Hamamatsu Photonics, http://www.hamamatsu.com/.

${ }^{10}$ A. Pietropaolo, C. Andreani, A. Filabozzi, R. Senesi, G. Gorini, E. Perelli Cippo, M. Tardocchi, N. J. Rhodes, and E. M. Schooneveld, J. Instr. 1, 04001 (2006).

${ }^{11}$ C. Andreani, G. Gorini, E. Perelli-Cippo, A. Pietropaolo, N. Rhodes, E. M. Schooneveld, R. Senesi, and M. Tardocchi, Appl. Phys. Lett. 75, 5454 (2004).

${ }^{12}$ A. Pietropaolo and R. Senesi, Nucl. Instrum. Methods Phys. Res. A 584,
377 (2008).

${ }^{13}$ F. Grazzi, M. Celli, S. Siano, and M. Zoppi, Nuovo Cimento Soc. Ital. Fis., C 30, 59 (2007).

${ }^{14}$ L. Batoli, M. Celli, F. Grazzi, S. Imberti, S. Siano, and M. Zoppi, La Metallurgia Italiana 9/2008, 33 (2008).

${ }^{15}$ M. Celli, D. Colognesi, F. Grazzi, and M. Zoppi, Not. Neutroni Luce Sincrotone 11, 6 (2006).

${ }^{16}$ Official website of ISIS Pulsed Neutron \& Muon Source, http:// www.isis.rl.ac.uk.

${ }^{17}$ M. Tardocchi, G. Gorini, A. Pietropaolo, C. Andreani, R. Senesi, N. Rhodes, and E. M. Schooneveld, Rev. Sci. Instrum. 75, 4880 (2004). 